ISSN 1518-3483

Licenciado sob uma Licença Creative Commons

\title{
Resiliencia en docentes: Una vía para mejorar capacidades de las poblaciones ante inundaciones agravadas por el cambio climático
}

\author{
Resilience in teachers: A way to improve capacities of \\ populations to face a flood aggravated by climate change
}

\section{Erick Cajigal-Molina, Ana-Lucía Maldonado-González, Edgar J. González-Gaudiano*}

Universidad Veracruzana, Xalapa, México

\section{Resumen}

Una posible alternativa para reducir la vulnerabilidad ante inundaciones es contribuir a desarrollar resiliencia comunitaria. Los docentes de nivel medio superior (bachillerato) pueden ser actores clave en las comunidades y por tanto promotores de acciones que propicien resiliencia. En este texto se describe la metodología utilizada y los resultados iniciales de un estudio dirigido a identificar la resiliencia individual y comunitaria en docentes de bachillerato en tres localidades de los municipios de Tlacotalpan, Cotaxtla y La Antigua en el estado de Veracruz, México. Las condiciones físicas y sociales en las localidades del estudio incrementan la fragilidad de su población y por tanto su vulnerabilidad a sufrir

ECM: Doctorante en Investigación Educativa, e-mail: cajigal1983@hotmail.com ALMG: Doctora, e-mail: anmaldonado@uv.mx

EJGG: Doctor, e-mail: egonzalezgaudiano@gmail.com 
daños por inundaciones, posiblemente agravadas por el cambio climático. Tratándose de un fenómeno complejo y global, no existe lugar donde sus efectos no lleguen. Aunque los principales responsables del cambio climático son los países denominados desarrollados son los países en desarrollo los que sufren en mayor medida sus impactos. Entre sus consecuencias, el aumento de la temperatura global ha incidido en la intensidad de los ciclones tropicales resultando en inundaciones con severos costos en municipios vulnerables como los incluidos en esta investigación.

Palavras-chave: Resiliencia. Cambio climático. Vulnerabilidad. Docentes. Veracruz.

\section{Abstract}

A way to reduce vulnerability face flooding can be to helping community to develop resilience. High school teachers could be key actors in communities, therefore promoters of actions that foster resilience. This paper describes the methodology and the first results of a study aimed at identifying individual and community resilience in high school teachers in three localities of the municipalities of Tlacotalpan, Cotaxtla and La Antigua in the state of Veracruz, Mexico. Physical and social conditions in the study context increase the fragility of their population, then their vulnerability to flood damage. These floods are possibly aggravated by climate change. There is no any place where the effects of this global phenomenon do not reach. Although the main responsible for climate change are the so-called developed countries, it is the developing countries that suffer most from their impacts. Among its consequences, the increase in global temperature has affected the intensity of tropical cyclones resulting in floods with severe costs in vulnerable municipalities, such as those included in this research.

Keywords: Resilience. Climate change. Vulnerability. Teachers. Veracruz. 


\section{Introdução}

La naturaleza compleja del cambio climático (CC) dificulta comprender todas sus implicaciones en nuestras vidas; no obstante, algunos fenómenos como los ciclones tropicales (CT) muestran evidencias crecientes en cuanto a intensidad y frecuencia que podrían ser vinculados con el CC (GLEIXNER, et al., 2013). En los últimos años se han experimentado por todo el mundo CT catastróficos, por ejemplo: Katrina (2005), Dean (2007), Irene (2011), Ingrid y Manuel (2013), Dolly (2014), Patricia (2015) y Earl (2016). Aún más, se ha registrado un aumento en la temperatura promedio global (IPCC, 2015), con evidencia proveniente de diversas agencias (NASA, NOAA y OMM) que consignan al año 2016 como el más caluroso desde 1880 , cuya temperatura global se situó 1,1 grados centígrados por arriba de la ocurrida en la era preindustrial y estuvo 0,07 grados más caliente que 2015; tal incremento ha sido causa de alarma mundial.

El aumento en la temperatura promedio global ha derivado en alteraciones del clima, reflejadas en fenómenos hidrometeorológicos de mayor intensidad tales como sequías y $\mathrm{CT}$, con sus consecuentes afectaciones a poblaciones expuestas. De 2005 a 2015 a nivel mundial "Los desastres, muchos de los cuales se ven exacerbados por el cambio climático, están aumentando su frecuencia e intensidad" (NACIONES UNIDAS, 2015, p. 10). En este mismo periodo, estos desastres alrededor del mundo han afectado a más de 700000 personas, 1.4 millones han resultado con heridas, más de 23 millones han quedado sin hogar, 144 millones han sido desplazadas y las pérdidas económicas superan los 1.3 billones de dólares (NACIONES UNIDAS, 2015).

En México, particularmente en el estado de Veracruz localizado en región intertropical, cada año se presenta la amenaza de CT. Se ha detectado que estos fenómenos muestran un incremento significativo en sus precipitaciones (CNIAN, 2012) y con ello en sus afectaciones. Las lluvias intensas y los CT han ocasionado inundaciones, decesos y perjuicios en los niveles sociales y económicos, así como severos daños en los ecosistemas en el estado. 
En este contexto, las afectaciones inmediatas por el impacto de eventos climáticos como los CT y sus consecuentes inundaciones en las comunidades son principalmente lesiones en la población, pérdidas de vidas y de bienes, daños e interrupción de los servicios básicos e infraestructura, desorganización social y alteraciones conductuales de los pobladores (BERMÚDEZ, 1993). Asimismo, a mediano y largo plazos las afectaciones frecuentes por inundaciones pueden ocasionar "disminución de los medios de subsistencia, impactando en la producción y en otros efectos económicos, esto puede provocar un proceso migratorio o desplazamientos de las poblaciones a otras regiones. También las familias pueden vivir efectos graves psicosociales graves" (TRAVIESO, 2012, p. 104).

Aunado a lo anterior, la recuperación varía entre las comunidades ya que la manera de responder ante las adversidades y su capacidad para sobreponerse después de los desastres no es la misma, toda vez que está asociada a su resiliencia. El IPCC (2015) define resiliencia como la capacidad de recuperación de los sistemas sociales, económicos y ambientales para hacer frente, responder o reorganizarse ante un evento peligroso, de manera que conserven su función esencial, su identidad y su estructura, al tiempo que se mantenga la capacidad de adaptación, aprendizaje y transformación.

El concepto de resiliencia ha sido utilizado en disciplinas como la física y la biología; se ha trasladado a las ciencias sociales y la psicología donde es empleado para explicar cómo algunas personas o grupos afectados pueden sobreponerse antes que otros a una adversidad similar. Desde lo social existen tres enfoques en las definiciones de resiliencia: estabilidad, recuperación y transformación (URIARTE, 2013); este estudio coincide con el enfoque de transformación que señala "la capacidad humana para enfrentar, sobreponerse y ser fortalecido por experiencias de adversidad" (HENDERSON, 2001, p. 20). En otras palabras, se refiere al aprendizaje de nuevas habilidades y a la transformación personal que sucede después de enfrentar un infortunio (RICHARDSON et al., 1990).

La resiliencia tiene dos senderos interpretativos. El primero hace referencia a las personas que nacen con capacidades resilientes; el segundo expresa que dichas capacidades pueden desarrollarse en determinadas situaciones a 
lo largo de la vida. En este segundo sendero la figura cercana de un adulto significativo, que promueva modelos de resolución pertinentes a los problemas, empatía e interés, ayuda al proceso de resiliencia. Tal figura puede encontrarse en los padres, familiares y educadores, entre otros (MELILLO, 2001).

En zonas frecuentemente afectadas por inundaciones como las que conforman nuestro estudio, los docentes de bachillerato pueden posicionarse como figuras significativas. Lo anterior al promover factores resilientes en sus alumnos, quienes posteriormente podrán promover esto mismo entre sus familiares, amistades y vecinos, desarrollando así capacidades a favor de la resiliencia comunitaria. Para lograr lo anterior, es necesario tener docentes resilientes.

De ahí que la investigación que origina este texto tiene como objetivo identificar la resiliencia individual y comunitaria en docentes de bachillerato en los municipios de Tlacotalpan, Cotaxtla y La Antigua. Esto con el fin de brindar un diagnóstico a ser considerado en acciones que contribuyan para mejorar las capacidades de prevención, recuperación y transformación comunitaria ante este tipo de eventos. Esos municipios han registrado diversas inundaciones; el último episodio con severos costos ocurrió en 2010 con los CT Karl y Matthew. El nivel de precipitación de Karl fue de $355 \mathrm{~mm}$, con vientos sostenidos de $185 \mathrm{~km} / \mathrm{h}$ y rachas de 230 $\mathrm{km} / \mathrm{h}$, fue catalogado como un huracán extremadamente peligroso, cobró la vida de 12 personas tan sólo en el estado de Veracruz (HERNÁNDEZ y ROSENGAUS, 2010) y dejó pérdidas que se calculan alrededor de los cinco mil millones de dólares (TEJEDA, 2012).

Tlacotalpan, se encuentra en la zona centro del estado y cuenta con un total de 13,284 habitantes (INEGI, 2010). En 2010 la cabecera municipal permaneció inundada en dos ocasiones: la primera a causa de Karl entre el 28 de agosto y 21 de septiembre. La segunda inundación provocada por las secuelas de Matthew, del 28 de septiembre al 4 de octubre (TEJEDA, 2012). Las labores para aminorar la inundación resultaron inadecuadas; por ejemplo, previo a la segunda inundación instalaron un muro de sacos de arena que fue rebasado por el río Papaloapan durante el 
impacto del CT Matthew, ese mismo muro impidió el regreso del agua al río derivando en inundación por siete días ${ }^{1}$.

Cotaxtla, municipio que se encuentra en la zona centro del estado de Veracruz con una población total de 19710 habitantes (INEGI, 2010), también vivió algo atípico en 2010 con el paso del huracán Karl. El río Cotaxtla en pocas horas elevó su caudal por más de 17 metros, llegando así a inundar la cabecera municipal y ocasionando graves afectaciones, inclusive hubo pérdidas humanas.

Finalmente, el municipio de La Antigua, que se ubica en la zona centro del estado de Veracruz y que cuenta con una población total de 25500 habitantes (INEGI, 2010), después de la inundación por el huracán Karl en 2010, algunas comunidades como San Pancho no lograron recuperarse y tuvieron que ser reubicadas a otras zonas; sin embargo, poco tiempo pasó para descubrir que el lugar donde se establecieron también padece inundaciones ${ }^{4}$.

En este contexto, resulta urgente priorizar la promoción de conductas resilientes en estas poblaciones y brindar elementos que contribuyan a reducir las afectaciones por inundaciones que ahí se viven.

\section{Metodología}

El estudio se realizó con técnicas propias de la investigación cuantitativa, diseñando y aplicando un instrumento titulado: encuesta resiliencia (Anexo 1). La encuesta es uno de los métodos más utilizados en la investigación social cuantitativa, por el cual se obtiene información mediante preguntas orales o escritas que se hacen directamente a la población o bien a su muestra (BRIONES, 1996).

La encuesta fue de tipo explicativo. Este tipo de encuestas como su nombre lo dice busca explicar un fenómeno mediante la asociación y análisis de variables (BRIONES, 1996). Este instrumento se conformó de

1 Información recuperada por los autores en actividades de trabajo de campo exploratorias y de reconocimiento de la zona. 
48 ítems, de los cuales los primeros 31 corresponden a la identificación de la resiliencia individual, los ítems 33 a 36 tienen el propósito de caracterizar a los participantes y los restantes están diseñados para identificar la resiliencia comunitaria ${ }^{2}$.

La encuesta fue piloteada con docentes de bachillerato de Juchique de Ferrer, Veracruz, municipio que también ha vivido episodios de inundaciones y deslaves por CT (CAJIGAL, 2014). El pilotaje reveló que era necesario modificar los ítems 32 y 34, además de hacer modificaciones en el cuadro de presentación. Con los datos obtenidos se realizó la prueba de confiabilidad Kuder Richardson, que es el equivalente a la prueba Alfa de Cronbach, pero para pruebas dicotómicas. Este tipo de pruebas brindan certeza sobre la asignación numérica que, en este caso, los docentes han dado a los ítems y sobre su homogeneidad (QUERO, 2010). El resultado fue 0.81 que se interpreta como alta confiabilidad (CAMPO-ARIAS y OVIEDO, 2008).

Cada uno de los ítems se desprendía de categorías fundamentadas teórica y empíricamente; por ejemplo la resiliencia individual después de una revisión bibliográfica (MUNIST, et al., 1998; MELILLO, 2001; SALAMANCA, 2009; GAMARRA, 2010) y la construcción del marco teórico-conceptual (RICHARDSON, et al., 1990; HENDERSON, 2001; SUÁREZ OJEDA, 2001; HENDERSON y MILSTEIN, 2003) se integró por un conjunto de cinco categorías denominadas: 1) Ambiente laboral; 2) En torno al Yo; 3) Familia; 4) Figura de adulto significativo y 5) Condiciones físicas y de salud (Tabla 1).

Por su parte, la resiliencia comunitaria se conforma de seis categorías derivadas de la revisión bibliográfica (GAMARRA, 2010), del marco teórico-conceptual (SUÁREZ OJEDA, 2001) y de actividades paralelas a esta investigación, en donde los autores también participaron. Las categorías son: 1) Autoestima colectiva; 2) Identidad cultural; 3) Honestidad colectiva; 4) Consuelo a partir de la experiencia de otros; 5) Humor social y 6) Instituciones significativas. En la Tabla 1 se muestra el propósito de cada una de las categorías

2 Existen ítems invertidos: 14, 27, 28 y 29, en los que la opción NO es respuesta positiva y opción Sí es respuesta negativa. El ítem 40 para el caso particular de este estudio fue invertido durante el análisis. 
para la resiliencia individual y comunitaria, así como el número de ítem que le corresponde en el instrumento y el tipo de respuesta asignado.

Tabla 1 - Descripción de la encuesta resiliencia

(continúa)

\begin{tabular}{|c|c|c|c|c|}
\hline Ítem(s) & $\begin{array}{l}\text { Tipo de } \\
\text { resiliencia }\end{array}$ & $\begin{array}{l}\text { Tipo de } \\
\text { respuesta }\end{array}$ & Categoría & Descripción de Categoría \\
\hline 1 a 5 & Individual & $\begin{array}{l}\text { Dicotómica: } \\
\text { Sí y No }\end{array}$ & $\begin{array}{l}\text { Ambiente } \\
\text { laboral }\end{array}$ & $\begin{array}{l}\text { Identifica los factores que favorecen la } \\
\text { resiliencia en los docentes desde sus } \\
\text { centros de trabajo. }\end{array}$ \\
\hline 6 a 17 & Individual & $\begin{array}{l}\text { Dicotómica: } \\
\text { Sí y No }\end{array}$ & $\begin{array}{l}\text { En torno } \\
\text { al Yo }\end{array}$ & $\begin{array}{l}\text { Se integra por cuatro elementos: Yo tengo } \\
\text { (remite a cuestiones de apoyo), Yo soy y Yo } \\
\text { estoy (se refiere a aspectos de autoestima } \\
\text { y personalidad) y Yo puedo (actitud). }\end{array}$ \\
\hline 18 a 21 & Individual & $\begin{array}{l}\text { Gradual: } \\
\text { Bajo, } \\
\text { Regular y } \\
\text { Alto }\end{array}$ & \multirow[t]{2}{*}{ Familia } & \multirow[t]{2}{*}{$\begin{array}{l}\text { Identifica el nivel de cohesión familiar. La } \\
\text { unión de los integrantes de la familia es } \\
\text { una condición para desarrollar resiliencia } \\
\text { (HENDERSON, 2001). }\end{array}$} \\
\hline 22 & Individual & Abierta & & \\
\hline 23 y 24 & Individual & $\begin{array}{l}\text { Dicotómica: } \\
\text { Sí y No }\end{array}$ & \multirow{2}{*}{$\begin{array}{l}\text { Figura de } \\
\text { Adulto } \\
\text { significativo }\end{array}$} & \multirow{2}{*}{$\begin{array}{l}\text { Un adulto cercano puede facilitar el } \\
\text { desarrollo de la resiliencia si mantiene } \\
\text { empatía o vínculos emocionales con } \\
\text { el adolescente, además de proyectar } \\
\text { modelos apropiados para la solución de } \\
\text { problemas cotidianos (MELILLO, 2001). }\end{array}$} \\
\hline 25 y 26 & Individual & $\begin{array}{l}\text { Dicotómica: } \\
\text { Bajo y Alto }\end{array}$ & & \\
\hline 27 a 31 & Individual & $\begin{array}{l}\text { Dicotómica: } \\
\text { Sí y No }\end{array}$ & $\begin{array}{l}\text { Condiciones } \\
\text { físicas y de } \\
\text { salud }\end{array}$ & $\begin{array}{l}\text { Identifica las condiciones físicas y de } \\
\text { salud de los docentes. En una situación } \\
\text { de adversidad las personas en mejores } \\
\text { condiciones tienen mayores posibilidades } \\
\text { de no ser afectadas. }\end{array}$ \\
\hline 32 & Comunitaria & $\begin{array}{l}\text { Dicotómica: } \\
\text { Seria y } \\
\text { Humor }\end{array}$ & $\begin{array}{l}\text { Humor } \\
\text { social }\end{array}$ & $\begin{array}{l}\text { Habilidad de expresar agradablemente } \\
\text { un hecho sombrío, de manera que sea } \\
\text { posible a través del relato, provocar un } \\
\text { efecto de tranquilidad ante la tragedia. } \\
\text { Suárez Ojeda ( } 2001, \text { p. } 74 \text { ) afirma que "el } \\
\text { humor es una estrategia de ajuste que } \\
\text { ayuda a una aceptación madura de la } \\
\text { desgracia común [...] favoreciendo la toma } \\
\text { de decisiones para resolverlo". }\end{array}$ \\
\hline 33 a 36 & \multicolumn{4}{|c|}{ No aplica. Datos para caracterizar participantes. } \\
\hline
\end{tabular}


Tabla 1 - Descripción de la encuesta resiliencia

(conclusión)

\begin{tabular}{|c|c|c|c|c|}
\hline 37 a 42 & Comunitaria & $\begin{array}{c}\text { Dicotómica: } \\
\text { Sí y No }\end{array}$ & $\begin{array}{l}\text { Identidad } \\
\text { cultural }\end{array}$ & $\begin{array}{l}\text { Pretende conocer la incorporación } \\
\text { de costumbres, valores, ideologías, } \\
\text { celebraciones, etc. Son las acciones } \\
\text { o prácticas que indican un sentido de } \\
\text { pertenencia. }\end{array}$ \\
\hline 43 y 44 & Comunitaria & $\begin{array}{c}\text { Dicotómica: } \\
\text { Bajo y Alto }\end{array}$ & $\begin{array}{l}\text { Honestidad } \\
\text { colectiva }\end{array}$ & $\begin{array}{l}\text { Identifica el sentir de un grupo que condena } \\
\text { la deshonestidad y valora la honestidad de } \\
\text { la función pública que desgasta los vínculos } \\
\text { sociales. “Nadie está dispuesto a ofrecer su } \\
\text { esfuerzo solidario si no confía en quienes } \\
\text { administran los recursos que se asignen a esa } \\
\text { reconstrucción” (SUÁREZ OJEDA, 2001, p. 76). }\end{array}$ \\
\hline 45 & Comunitaria & $\begin{array}{l}\text { Dicotómica: } \\
\text { Sí y No; } \\
\text { respuesta } \\
\text { abierta }\end{array}$ & $\begin{array}{l}\text { Consuelo a } \\
\text { partir de la } \\
\text { experiencia } \\
\text { de otros }\end{array}$ & $\begin{array}{l}\text { Son las prácticas de reflexión sobre el grado } \\
\text { de daño sufrido y comparado éste con el } \\
\text { sufrido por otras personas mayormente } \\
\text { afectadas. Pensarse como personas } \\
\text { afortunadas por recibir menos afectaciones } \\
\text { que otras, proporciona un sentimiento de } \\
\text { optimismo que impulsa a la activación de } \\
\text { fortalezas para enfrentar, superar e incluso } \\
\text { aprender de la adversidad. }\end{array}$ \\
\hline Ítem(s) & $\begin{array}{l}\text { Tipo de } \\
\text { resiliencia }\end{array}$ & $\begin{array}{l}\text { Tipo de } \\
\text { respuesta }\end{array}$ & Categoría & Descripción de Categoría \\
\hline 46 & Comunitaria & $\begin{array}{l}\text { Dicotómica: } \\
\text { Sí y No }\end{array}$ & \multirow{2}{*}{$\begin{array}{l}\text { Instituciones } \\
\text { significativas }\end{array}$} & \multirow{2}{*}{$\begin{array}{l}\text { Se interesa en conocer las instituciones } \\
\text { en las cuales las personas han depositado } \\
\text { su confianza. Estas instituciones tienen } \\
\text { un papel determinante, su poder impacta } \\
\text { directamente en el quehacer de las } \\
\text { comunidades. }\end{array}$} \\
\hline 47 & Comunitaria & Abierta & & \\
\hline 48 & Comunitaria & $\begin{array}{c}\text { Dicotómica: } \\
\text { Orgullo e } \\
\text { indiferente }\end{array}$ & $\begin{array}{l}\text { Autoestima } \\
\text { colectiva }\end{array}$ & $\begin{array}{l}\text { Pretende identificar el orgullo por la } \\
\text { comunidad, el sentido de pertenencia y de } \\
\text { satisfacción hacia el lugar donde se vive, } \\
\text { "aquellas ciudades o localidades en las } \\
\text { que se observa una elevada autoestima } \\
\text { colectiva tengan mayor capacidad de } \\
\text { recuperación frente a las adversidades" } \\
\text { (SUÁREZ OJEDA, 2001, p. 72). }\end{array}$ \\
\hline
\end{tabular}

El instrumento se aplicó a una muestra representativa de la población de docentes de los bachilleratos públicos de los tres municipios. El tamaño de la muestra se determinó por el nivel de confianza del estudio que es de $90 \%$, con margen de error de $10 \%$ y una distribución de respuestas de $50 \%$. 
Se logró la participación de todos los docentes que la muestra establecía. En el bachillerato Agustín Yáñez del municipio de La Antigua participaron 28 docentes; en Tlacotalpan en el bachillerato Avelino Bolaños participaron 18 docentes y en el Centro de Bachillerato Tecnológico Industrial y de Servicios 35 (CBTIS 35) del mismo municipio 20 docentes; finalmente, en el municipio de Cotaxtla en el Telebachillerato participaron 9 docentes. En total fueron 75 docentes participantes, que corresponden al $76 \%$ de la población total de docentes de los tres municipios. Cabe hacer énfasis que los cuatro planteles mencionados son los únicos bachilleratos públicos en los municipios de estudio.

La distribución por género de los docentes participantes correspondió a $51 \%$ mujeres y $49 \%$ hombres, cuya edad promedio es de 40 años. Cuentan con formaciones variadas, aunque la licenciatura que más se registra es pedagogía (10 docentes). En este sentido, siete docentes cuentan con posgrado; de estos, seis son maestrías relacionadas con la educación. Por último, en cuanto a la trayectoria laboral de los participantes tienen un promedio de 10 años en servicio.

La aplicación de la encuesta se realizó en cuatro días (no consecutivos), uno por bachillerato. La dinámica de aplicación consistió en una presentación por parte del aplicador, la facilitación del instrumento y la orientación para responder, en caso de necesitarla. El aplicador fue siempre el mismo. La duración de aplicación osciló entre 11 y 25 minutos; no hubo contratiempos, en todos los casos se mostró disposición de los docentes para participar. Cabe destacar que previo a la aplicación del instrumento se habían realizado varias visitas para explorar el contexto y las condiciones de estudio. Además de esta etapa para investigar sobre la resiliencia, se tuvieron otras visitas más para investigar sobre aspectos de la misma investigación relacionados con la vulnerabilidad, lo que no es posible incluir en este artículo.

Se realizó la captura de la información en el programa estadístico SPSS y se realizó la prueba de confiabilidad Kuder Richardson en Excel. Los resultados en todos los casos son confiables oscilando entre $0.80 \mathrm{y}$ 0.86 . Finalmente, se realizaron tablas de frecuencia de cada uno de los ítems y se conjuntaron por categorías para evidenciar el nivel de resiliencia individual y comunitaria de los docentes de bachillerato. 


\section{Resultados y conclusiones}

Los resultados sobre la resiliencia individual indican que existen categorías altamente fortalecidas como: En torno al Yo, Familia y Figura de adulto significativo. Sin embargo, se encuentran como áreas de oportunidad las categorías: Ambiente laboral y Condiciones físicas y de salud.

En este sentido, la categoría En torno al Yo representada en la encuesta por un conjunto de 12 ítems, del 6 al 17, registró porcentajes superiores en todos los casos al 77.8\%; por su parte la categoría Familia, identificada con los ítems 18 a 22 de la encuesta, en todos los casos resultan positivos con un promedio de respuestas positivas de $79.9 \%$; y la categoría Figura de adulto significativo, representada por los ítems 23 a 26 de la encuesta, obtuvo en todos los casos porcentajes superiores al $80 \%$. Lo que significa que la mayoría de categorías contribuyen a la construcción de la resiliencia.

Como área de oportunidad se encuentra la categoría Ambiente laboral. Esta categoría mostró en tres casos (Telebachillerato de Cotaxtla, Agustín Yáñez de La Antigua y en el CBTIS 35 de Tlacotalpan) resultados negativos y en un cuarto caso (Avelino Bolaños de Tlacotalpan) los resultados con un porcentaje bajo logran posicionarse en positivo, de ahí que se considere que los resultados no favorecen el desarrollo de la resiliencia. Esta categoría, revela puntualmente que no existen oportunidades para que los docentes puedan capacitarse y mejorar su práctica educativa. Asimismo, los participantes señalaron en todos los casos que no hay reconocimiento social del papel que cumplen los docentes.

La categoría Condiciones físicas y de salud también es considerada área de oportunidad. Aquí encontramos que el sobrepeso corporal, presente en la mayoría de los docentes, es la problemática central que no permitió posicionar a esta categoría de manera positiva. Lo anterior debido a que, al preguntarles sobre su alimentación, la mayoría de los docentes participantes expresa no tener algún cuidado especial y tampoco realiza actividades deportivas con frecuencia. Por lo tanto, en una situación de adversidad se incrementan las posibilidades de ser afectados, debido a sus condiciones físicas y de salud. 
Por otro lado, en lo que corresponde a la resiliencia comunitaria las categorías: Humor social, Identidad cultural, Consuelo a partir de la experiencia, Autoestima colectiva e Instituciones significativas revelan una aportación positiva; la categoría que requiere atención para considerarse en el desarrollo de la resiliencia comunitaria es Honestidad colectiva.

En este sentido, la categoría Humor social, que fue identificada con el ítem 32 de la encuesta, resultó altamente positiva con porcentajes superiores a $88 \%$ y fue confirmada en diferentes momentos que los autores interactuaron con los docentes participantes en el trabajo de campo. Esta característica les ha ayudado a salir adelante ante las adversidades como en las inundaciones que han experimentado, debido a que puede provocar efectos de tranquilidad y optimismo ante la tragedia para activar sus fortalezas y salir adelante.

Otra categoría que obtuvo una valoración positiva fue Identidad cultural. Esta fue identificada con seis ítems representados del 37 al 42 de la encuesta. Es importante decir que el análisis de los datos obtenidos por el ítem 40 determinó su sentido; aquí se pretendía conocer las acciones o prácticas, valores, ideologías y celebraciones que indican un sentido de pertenencia y los resultados obtenidos indican que las comunidades rechazan participar en actividades políticas, tal rechazo es una acción colectiva que se interpreta como un signo de identidad cultural de la población, de ahí que se invierta. Este rechazo puede estar asociado a la categoría Honestidad colectiva, la cual se explica más adelante.

Los resultados sobre la categoría Consuelo a partir de la experiencia, fueron positivos para Tlacotalpan y La Antigua; es decir, que los docentes perciben que su comunidad no fue tan afectada como otras, contrariamente a los docentes de Cotaxtla quienes señalan que la comunidad fue la más afectada. El resultado anterior es congruente en sí mismo. En los ítems 33 y 34 de la encuesta de resiliencia se indaga sobre si el docente reside en esa comunidad o si se traslada; los resultados obtenidos indican que ocho de los nueve docentes que ahí laboran no viven en Cotaxtla y el que sí vive en la comunidad considera que hubo otras comunidades más afectadas. Este resultado deja ver que el sentimiento de pertenencia hacía la comunidad donde se reside no permite describirla como la más 
afectada; en otras palabras, el orgullo por el lugar donde tienen sus hogares les impide verse a sí mismos como vulnerables.

Por su parte, la categoría Autoestima colectiva identificó el orgullo y la satisfacción hacia el lugar donde los docentes laboran. En todos los casos el resultado fue positivo, aunque Cotaxtla registró el porcentaje más bajo. Este resultado permite inferir que existe correlación entre la presente categoría y la de Consuelo a partir de la experiencia de otros; en Cotaxtla ocho de nueve docentes no residen ahí, se trasladan periódicamente, por tanto el sentimiento de pertenencia es mayor hacía el lugar donde se reside más no donde se labora.

En cuanto a la categoría Instituciones significativas, representadas en los ítems 46 y 47 de la encuesta, se pudo observar que los docentes perciben a sus planteles educativos como significativos en su comunidad, aunque también se reconocen otras instituciones significativas. Los resultados señalan en todos los casos hacia otras instituciones educativas de diversos niveles, desde primaria hasta superior, y hacia las iglesias católicas. El percibirse como institución significativa aporta directamente a la resiliencia, pero además si se establecieran vínculos con las otras instituciones que los docentes han señalado como significativas se incrementarían las posibilidades de desarrollar la resiliencia comunitaria.

Finalmente, la categoría que se considera como área de oportunidad por no aportar al desarrollo de la resiliencia comunitaria en este momento es: Honestidad colectiva; esta categoría se identificó con los ítems 43 y 44 de la encuesta. La razón de no contribuir se debe a que en todos los casos se valoró negativamente la honestidad de las autoridades estatales. Quizás esta valoración contribuye al bajo interés detectado por participar en actividades políticas, mismo que se refleja dentro de la categoría Identidad cultural.

Tales resultados ante las inundaciones por CT cobran relevancia al pretender fundamentar estrategias que permitan a los pobladores desarrollar capacidades resilientes para resistir, recuperarse o incluso transformarse frente a este tipo de adversidades, iniciando con los docentes de bachillerato, proyectándose a sus alumnos y estos últimos compartiendo con la comunidad en general. 
Los docentes resilientes pueden posicionarse como adultos significativos para sus alumnos, proveyendo modelos de resolución y liderazgo, así como formación específica para reducir la vulnerabilidad ante las inundaciones que enfrentan sus comunidades. Para esto, en principio se considera necesario establecer colaborativamente estrategias para mejorar las categorías que no están aportando al desarrollo de la resiliencia en los docentes. Posteriormente, involucrar a los docentes en una formación desde un enfoque complejo e interdisciplinario que les permita comprender y atender problemáticas ambientales con acciones pertinentes desde los espacios escolares. Todo ello, en conjunto, podrá contribuir a que los docentes, alumnos y comunidad en general emprendan acciones para enfrentar adversidades y disminuir las afectaciones por inundaciones derivadas de CT agravados por el CC, sin depender tanto de las capacidades de gestión de las autoridades locales, las cuales en este tipo de situaciones han sido rebasadas (GONZÁLEZ GAUDIANO y MALDONADO, 2017).

\section{Referencias}

BERMÚDEZ, M. Vulnerabilidad social y organización ante los desastres naturales. Revista de Ciencias Sociales, n. 62, p. 131-141, 1993.

BRIONES, G. Metodología de la investigación cuantitativa en las ciencias sociales. Colombia: Instituto Colombiano para el Fomento de la Educación Superior, ICFES, 1996.

CAJIGAL, E. Vulnerabilidad y resiliencia social ante inundaciones derivadas de ciclones tropicales en tres municipios de Veracruz. Un estudio con docentes de nivel bachillerato. Tesis doctoral en Investigación Educativa, Universidad Veracruzana, con la dirección de A. L. MALDONADO, en proceso.

CAJIGAL, E. Las creencias epistemológicas en docentes de educación primaria y su impacto en la construcción de conocimiento sobre el cambio climático. (Tesis) Maestría - Universidad Veracruzana, Veracruz, México, 2014. 
CAMPO-ARIAS, A. Y OVIEDO, H. C. Propiedades Psicométricas de una Escala: la Consistencia Interna. Revista Salud Pública, v. 10, n. 5, 2008.

CNIAN. Cambio climático. Evidencias, impactos y opciones. Respuestas a preguntas comunes sobre la ciencia del cambio climático. Estados Unidos: Consejo Nacional de Investigaciones de las Academias Nacionales, 2012.

CONAPO. Índice de marginación por entidad federativa y municipio 2010. Recuperado de: <http://www.conapo.gob.mx/es/CONAPO/Indices_de_Marginacion_2010_por_entidad_federativa_y_municipio $>$.Acceso en:10 mar. 2017.

GAMARRA, J. Resiliencia social y cambio en comunidades campesinas afectadas por conflicto armado interno: el caso de las comunidades de Incaraccay y Tanquihua en la provincia de Cangallo, Ayacucho. Perú: Instituto Peruano de Educación en Derechos Humanos y la Paz, 2010.

GLEIXNER, S., et al. An inter-hemispheric comparison of the tropical storm response to global warming. Climate Dynamics, n. 42, p. 2147-2157, 2013.

GONZÁLEZ GAUDIANO, E. J. Y MALDONADO A. L. Amenazas y riesgos climáticos en poblaciones vulnerables. El papel de la educación en la resiliencia comunitaria. Teoría Educativa, v. 29, n. 1, p. 273-294, 2017.

HERNÁNDEZ, A. Y ROSENGAUS, M. Análisis de la temporada de ciclones tropicales 2010. México: Comisión Nacional del Agua y Servicio Meteorológico Nacional, 2010.

HENDERSON, N. Introducción. Nuevas tendencias en resiliencia. En: A. Melillo y E. N. Suárez Ojeda (Compiladores). Resiliencia descubriendo las propias fortalezas. Argentina: Paidós, 2001. p. 19-30.

HENDERSON, N.; MILSTEIN, M. Resiliencia en la escuela. Argentina: Editorial Paidós, 2003.

INEGI. Información Nacional, por Entidad Federativa, 2010. Recuperado de: <http://www.beta.inegi.org.mx/app/areasgeograficas/?ag=30>. Acceso en: 11 abr. 2017. 
IPCC. Synthesis Report. Contribution of Working Groups I, II and III to the Fifth Assessment Report of the Intergovernmental Panel on Climate Change. Suiza: Grupo Intergubernamental de Expertos sobre el Cambio Climático, 2015.

NACIONES UNIDAS. Marco de Sendai para la Reducción del Riesgo de Desastre 2015-2030. Suiza, 2015. Recuperado de: <http://www.unisdr.org/files/43291_ spanishsendaiframeworkfordisasterri.pdf >. Acceso en: 1 jun. 2017.

MELILLO, A. C. Prefacio. En: MELILLO, A.; SUAREZ OJEDA, E. (Compiladores). Resiliencia descubriendo las propias fortalezas. Argentina: Paidós, 2001. p. 15-18.

MUNIST, M., et al. (1998). Manual de identificación y promoción de la resiliencia en los niños y adolescentes. Estados Unidos: Organización Panamericana de la Salud, 1998. QUERO, M. Confiabilidad y coeficiente Alfa de Cronbach. TELOS. Revista de Estudios Interdisciplinarios en Ciencias Sociales, v. 12, n. 2, p. 248-252, 2010.

RICHARDSON, G. E. et al. The Resiliency Model. Health and Education, v. 21, n. 6, p. 33-39, 1990.

SALAMANCA, L. A. Estudio de resiliencia en desastres naturales en seis barrios de la ciudad de La Paz, Bolivia. En: Estrategia Internacional para la Reducción de los Desastres (Coordinador), La gestión del riesgo urbano en América Latina: recopilación de artículos, 2009. p. 205-222.

SUÁREZ OJEDA, E. N. Una concepción latinoamericana: la resiliencia comunitaria. En: MELILLO, A.; SUAREZ OJEDA, E. N. (Compiladores) Resiliencia descubriendo las propias fortalezas. Argentina: Paidós, 2001. p. 67-82.

TEJEDA, A. Introducción. Apuntes corográficos de las inundaciones en el estado de Veracruz. En: TEJEDA, A. (Ed) Las inundaciones de 2010 en Veracruz. Memoria social y medio físico. México: Consejo Veracruzano de Investigación Científica y Desarrollo Tecnológico,2012. p. 21-42. 
TRAVIESO, A, C. Aplicación del modelo Presión-Estado-Respuesta al análisis de riesgos por inundaciones en la cuenca del río Nautla. En: TEJEDA, A.; ARENAS, V. (Coordinadores). Las inundaciones de 2010 en Veracruz. La biósfera, escenarios y herramientas. México: Universidad Veracruzana, 2012. p. 91-124.

URIARTE, J. La perspectiva comunitaria de la resiliencia. Psicología Política, n, 47, p. 7-18, 2013.

Recibido: 05/07/2017

Received: 07/05/2017

Aprobado: 31/07/2017

Approved: 07/31/2017 


\section{ANEXO 1}

Estimado(a) Maestro(a), esta es una encuesta realizada por el Doctorante Erick Cajigal Molina del Instituto de Investigaciones en Educación de la Universidad Veracruzana. Si usted decide participar con esta investigación puede estar seguro de que la información que proporcione será utilizada para brindar elementos que sirvan para planificar estrategias en beneficio de esta comunidad. Esta es una encuesta de carácter voluntario, confidencial y anónimo. De esto último, sepa usted que no solicitamos nombre del participante, sin embargo sí solicitamos un alto nivel de confianza y por ende transparencia en sus respuestas.

Docente de semestre(s)

Edad

Años de servicio

Sexo: M F

Formación (licenciatura y)

\section{Seleccione una opción}

En su escuela...

\begin{tabular}{|l|c|c|}
\hline $\begin{array}{l}\text { 1.- Considera que las reglas del bachillerato se aplican para todos los docentes } \\
\text { (puntualidad, permisos, horarios, etc.) }\end{array}$ & Sí & No \\
\hline $\begin{array}{l}\text { 2.- Considera que existe un buen ambiente de trabajo entre los docentes } \\
\text { 3.- Si decidiera capacitarse (tomar otros cursos, un posgrado, etc.) para mejorar su } \\
\text { práctica educativa, considera que sus superiores le darían la oportunidad y apoyo } \\
\text { para hacerlo }\end{array}$ & Sí & No \\
\hline $\begin{array}{l}\text { 4.- Considera que el papel de un docente es reconocido y valorado por la } \\
\text { comunidad }\end{array}$ & Sí & No \\
\hline $\begin{array}{l}\text { 5.- Ha propuesto o realizado proyectos que están fuera de los planes de estudio, } \\
\text { pero sabe que dejarán algo positivo en sus estudiantes } \\
\text { Si su respuesta fue Sí, podría mencionarnos algunos: }\end{array}$ & Sí & No \\
\hline
\end{tabular}

En su escuela, en su familia, con las amistades y/o en la comunidad...

\begin{tabular}{|l|c|c|}
\hline 6.- Se considera una persona altamente estimada por otros & Sí & No \\
\hline $\begin{array}{l}\text { 7.- } \text { Frente a un problema, considera que usted es parte de la solución, es decir } \\
\text { considera que puede ayudar en algo }\end{array}$ & Sí & No \\
\hline $\begin{array}{l}\text { 8.- } \text { Si se encuentra ante una adversidad o un desastre, considera que cuenta con } \\
\text { personas cercanas que pueden ayudarle }\end{array}$ & Sí & No \\
\hline $\begin{array}{l}\text { 9.- } \text { Considera que alrededor de usted hay personas de las que aprende conductas } \\
\text { correctas o que son un modelo a seguir }\end{array}$ & Sí & No \\
\hline $\begin{array}{l}\text { 10.- Considera que es una persona por la que sienten aprecio y cariño } \\
\text { 11.- Cuando hace algo bueno por los demás se siente feliz }\end{array}$ & So Sí & No \\
\hline 12.- Cuando algo no le parece pertinente o no le gusta, lo hace saber & Sí & No \\
\hline 13.- Cree que todo lo que hace tiene muchas posibilidades de salir bien & Sí & No \\
\hline
\end{tabular}

Rev. Diálogo Educ., Curitiba, v. 17, n. 55, p. 1445-1464, out./dez. 2017 


\begin{tabular}{|l|c|c|}
\hline 14.- Si algo sale mal y sabe que es su culpa, trata de que otros se responsabilicen & Sí & No \\
\hline 15.- Si tiene algún problema o inquietud lo comparte con otros & Sí & No \\
\hline $\begin{array}{l}\text { 16.- Cuando hay problemas busca resolverlos de inmediato } \\
\text { 17.- Si existe un problema, adversidad o desastre, elige participar en las actividades } \\
\text { de recuperación }\end{array}$ & Sí & No \\
\hline
\end{tabular}

En su familia...

\begin{tabular}{|c|c|c|c|}
\hline $\begin{array}{l}\text { 18.- Desde su perspectiva, qué nivel de comunicación tiene su } \\
\text { familia }\end{array}$ & Bajo & Regular & Alto \\
\hline 19.- Considera que el nivel de unión de su familia es: & Bajo & Regular & Alto \\
\hline $\begin{array}{l}\text { 20.- En cuanto a las actividades cotidianas, el nivel de cooperación } \\
\text { de su familia es: }\end{array}$ & Bajo & Regular & Alto \\
\hline $\begin{array}{l}\text { 21.- En una situación adversa o desastre y de resultar afectados, } \\
\text { qué nivel de cooperación tendría su familia para salir adelante: }\end{array}$ & Bajo & Regular & Alto \\
\hline \multicolumn{4}{|c|}{$\begin{array}{l}\text { 22.- En una situación adversa o desastre y de resultar afectados, quién o quiénes de su familia } \\
\text { serían las figuras que impulsarían salir adelante nuevamente }\end{array}$} \\
\hline
\end{tabular}

Como docente...

\begin{tabular}{|l|c|c|}
\hline 23.- Considera que apoya en la resolución de los problemas de sus estudiantes & Sí & No \\
\hline 24.- Considera que proyecta modelos de conducta que los estudiantes adoptan & Sí & No \\
\hline 25.- El nivel de aceptación que tiene en los estudiantes es: & Bajo & Alto \\
\hline 26.- El nivel de aceptación que tiene en los padres de familia es: & Bajo & Alto \\
\hline
\end{tabular}

Usted...

\begin{tabular}{|l|c|c|}
\hline 27.- Padece de alguna enfermedad crónica & Sí & No \\
\hline 28.- Se enferma constantemente de gripa, tos, etc. (enfermedades comunes) & Sí & No \\
\hline 29.- Considera que tiene sobrepeso & Sí & No \\
\hline 30.- Tiene un cuidado especial en su alimentación & Sí & No \\
\hline 31.- Realiza alguna actividad deportiva con frecuencia & Sí & No \\
\hline $\begin{array}{l}\text { 32.- Considera que es una persona generalmente con mal humor o } \\
\text { generalmente con humor }\end{array}$ & Humor & $\begin{array}{c}\text { Mal } \\
\text { Humor }\end{array}$ \\
\hline
\end{tabular}

\section{Por favor conteste lo siguiente.}

33.- ¿Vive en esta comunidad? (si su respuesta fue Sí, pase a la pregunta 35)

34.- ¿Se traslada diariamente a esta comunidad o con qué frecuencia?

\begin{tabular}{|l|c|c|}
\hline $\begin{array}{l}\text { 35.- Le gustaría trabajar en otra comunidad } \\
\text { Si respondió Sí, indique dónde__í }\end{array}$ & No \\
\hline $\begin{array}{l}\text { 36.- Le gustaría tener su hogar en otra comunidad } \\
\text { Si respondió Sí, indique dónde_ }\end{array}$ & Sí & No \\
\hline
\end{tabular}




\begin{tabular}{|c|c|c|c|}
\hline \multicolumn{2}{|l|}{ 37.- Participa en las costumbres locales de esta comunidad } & Sí & No \\
\hline \multicolumn{2}{|l|}{ 38.- Celebra las fiestas locales de esta comunidad } & Sí & No \\
\hline \multicolumn{2}{|l|}{ 39.- Participa en actividades religiosas (de cualquier religión) de esta comunidad } & Sí & No \\
\hline \multicolumn{2}{|l|}{ 40.- Participa o ha participado en actividades políticas de esta comunidad } & Sí & No \\
\hline \multicolumn{2}{|l|}{ 41.- Participa o ha participado en actividades deportivas de esta comunidad } & Sí & No \\
\hline \multicolumn{2}{|l|}{$\begin{array}{l}\text { 42.- Participa o ha participado en actividades de entretenimiento en esta } \\
\text { comunidad }\end{array}$} & Sí & No \\
\hline \multicolumn{2}{|l|}{ 43.- Que nivel de honestidad tienen las autoridades de esta comunidad } & Bajo & Alto \\
\hline \multicolumn{2}{|l|}{ 44.- Que nivel de honestidad tienen los vecinos de esta comunidad } & Bajo & Alto \\
\hline \multicolumn{2}{|l|}{$\begin{array}{l}\text { 45.- Esta comunidad se ha visto afectada por inundaciones, pero considera que } \\
\text { otras comunidades han sido más afectadas que ésta: } \\
\text { Sí su respuesta fue Sí, podría mencionar qué comunidades }\end{array}$} & Sí & No \\
\hline \multicolumn{2}{|l|}{ Por qué cree que fueron más afectadas } & & \\
\hline 46.- Considera que su escuela es significativa para la comunidad & & Sí & No \\
\hline \multicolumn{4}{|c|}{$\begin{array}{l}\text { 47- ¿Qué otras instituciones son significativas en la comunidad? (educativas, religiosas, gobierno, } \\
\text { etc.) }\end{array}$} \\
\hline $\begin{array}{l}\text { 48.- Siente orgullo por pertenecer a esta comunidad o le es indiferente } \\
\text { esta apreciación }\end{array}$ & Orgullo & Indife & ente \\
\hline
\end{tabular}

Gracias por participar en esta encuesta.

Fuente: Cajigal (en proceso). 